\title{
Lipid Accumulation Product Is More Related to Insulin Resistance than the Visceral Adiposity Index in the Maracaibo City Population, Venezuela
}

\author{
Valmore Bermúdez $\mathbb{D},{ }^{1}$ Juan Salazar $\mathbb{D}^{\mathrm{D}},{ }^{2}$ Jorge Fuenmayor ${ }^{\mathbb{D}},{ }^{2}$ Manuel Nava $\left(\mathbb{D},{ }^{2}\right.$ \\ Ángel Ortega $\left(\mathbb{1},{ }^{2}\right.$ Pablo Duran $\left(\mathbb{1},{ }^{2}\right.$ Milagros Rojas $\left(\mathbb{D},{ }^{2}\right.$ Roberto Añez $(\mathbb{1}),{ }^{3}$ \\ Alejandra Rivas-Montenegro, ${ }^{3}$ Lissé Angarita $\mathbb{D}^{4},{ }^{4}$ Maricarmen Chacín $\left(\mathbb{D},{ }^{1}\right.$ \\ Clímaco Cano $\left(\mathbb{D},{ }^{2}\right.$ Manuel Velasco $(\mathbb{D}){ }^{5}$ and Joselyn Rojas $\mathbb{D i D}^{6}$ \\ ${ }^{1}$ Universidad Simón Bolívar, Facultad de Ciencias de La Salud, Barranquilla, Colombia \\ ${ }^{2}$ Endocrine and Metabolic Diseases Research Center, School of Medicine, University of Zulia, Maracaibo, Venezuela \\ ${ }^{3}$ Department of Endocrine and Nutrition, Gregorio Marañón General University Hospital, Madrid, Spain \\ ${ }^{4}$ Universidad Andres Bello, Carrera de Nutrición, Concepción, Chile \\ ${ }^{5}$ Universidad Central de Venezuela, Escuela de Medicina José María Vargas, Caracas, Venezuela \\ ${ }^{6}$ Division of Pulmonary and Critical Care Medicine, Brigham and Women's Hospital and Harvard Medical School, Boston, \\ MA 02115, USA
}

Correspondence should be addressed to Juan Salazar; juanjsv18@hotmail.com

Received 5 January 2021; Accepted 30 May 2021; Published 7 June 2021

Academic Editor: Nunzio Velotti

Copyright (c) 2021 Valmore Bermúdez et al. This is an open access article distributed under the Creative Commons Attribution License, which permits unrestricted use, distribution, and reproduction in any medium, provided the original work is properly cited.

\begin{abstract}
Background. Visceral adiposity is related to insulin resistance (IR), a metabolic state considered as a risk factor for other cardiometabolic diseases. In that matter, mathematical indexes such as the visceral adiposity index (VAI) and the lipid accumulation product (LAP) could indirectly assess IR based on visceral adiposity. Objective. To evaluate the association and diagnostic accuracy of VAI and LAP to diagnose IR in the adult population of Maracaibo city. Methods. This is a cross-sectional descriptive study with multistage sampling. Receiver operating characteristic (ROC) curves were built to determine VAI and LAP cutoff points to predict IR. A set of logistic regression models was constructed according to sociodemographic, psychobiologic, and metabolic variables. Results. 1818 subjects were evaluated (51.4\% women). The area under the curve (AUC) values for LAP and VAI were $0.689(0.665-0.714)$ and $0.645(0.619-0.670)$, respectively. Both indexes showed a higher IR risk in the upper tertile in bivariate analysis. However, in the logistic regression analysis for the IR risk, only the 2nd (OR: $1.91 ; 95 \%$ CI: $1.37-2.65 ; p<0.01$ ) and 3rd (OR: 5.40; 95\% CI: 3.48-8.39; $p<0.01$ ) LAP tertiles showed a significant increase. This behaviour was also observed after adjusting for hs-C-reactive protein (hs-CPR). Conclusion. Although both indexes show a low predictive capacity in individuals with IR in the Maracaibo city population, the LAP index was more strongly associated with IR.
\end{abstract}

\section{Introduction}

Insulin resistance (IR) is a metabolic state in which insulindependent tissues progressively lose their sensitivity to this hormone. This phenomenon leads to metabolic imbalance and hyperinsulinemia [1]. Measuring IR precisely allows for the identification of individuals at risk of developing pathologies associated with this metabolic scenario, such as metabolic syndrome (MS), type 2 diabetes mellitus (T2DM), or polycystic ovarian syndrome (PCOS). Therefore, it allows for the implementation of timely and specific interventions [2]. The hyperinsulinemic-euglycemic clamp is considered the gold standard for the determination of IR. However, it is costly, and it requires time and trained personnel, especially 
for large-scale epidemiologic studies [3]. For this reason, more practical and accessible alternatives have been proposed, such as the homeostatic model assessment (HOMA) to evaluate IR, the quantitative insulin check index (QUICKI), and the Matsuda index for clinical use. Meanwhile, McAuley, Avignon, and Stumvoll indexes are more appropriate for epidemiologic and research studies [4].

The visceral adiposity index (VAI) represents an empiric sex-specific model proposed as a substitute marker of dysfunction and distribution of adipose tissue and its independent correlation with cardiometabolic risk. Recent research has determined a close relationship between the use of VAI and peripheral glycemia, IR, and T2DM $[5,6]$. On the contrary, the lipid accumulation product (LAP) expresses a constant adipose risk function associated with the development of cardiovascular disease and mortality in adults. It exhibits a predictive capacity to identify subjects at risk of presenting cardiovascular events as the age increased, which was observed more strongly in men [7]. Both indexes have been initially proposed as simple and easy tests to evaluate visceral adipose tissue dysfunction and cardiometabolic risk [8].

Furthermore, these indexes have proven to be valuable tools in identifying prediabetes, T2DM [9], and MS in patients with chronic kidney disease. Noticeably, LAP is superior in both women and men [10]. On the contrary, both VAI and LAP have proven to be promising markers to identify IR and cardiometabolic risk in obese and nonobese women with PCOS [11]. There is no consensus regarding the clinical index that shows a better discriminatory capacity to determine IR and cardiometabolic risk. Therefore, this study aimed to evaluate the association and diagnostic accuracy of VAI and LAP in diagnosing IR in the adult population from Maracaibo city.

\section{Materials and Methods}

2.1. Study Design and Selection of Subjects. The Maracaibo City Metabolic Syndrome Prevalence Study (MMSPS) was a cross-sectional, descriptive study performed in Maracaibo city, Venezuela. The methodology and main results of the study have been previously reported $[12,13]$. The subjects without insulin determination, type 2 and type 1 diabetes mellitus patients, and polycystic ovary syndrome patients were excluded in this secondary analysis; therefore, a total of 1818 subjects were evaluated. This study was approved by the Endocrine and Metabolic Diseases Research Center's Bioethics Committee (approval number: BEC-006-0305). All participants signed a written informed consent form before being interviewed and examined by a trained team.

2.2. Clinical Evaluation. All subjects included in the study went through a medical examination performed by trained personnel to obtain a complete clinical record. During the anamnesis, information about the past medical and family history of endocrine and metabolic disorders was collected. This record included age, race, working status, education, and socioeconomic status using the Graffar scale modified by Mendez-Castellano to evaluate the last aspect [14].
Subjects were asked about smoking habits and their duration, and they were categorized as current smokers, former smokers, and nonsmokers [15]. Physical activity was evaluated by the International Physical Activity Questionnaire [16]. For alcohol consumption, any subject that drank $\geq 1$ gram daily was considered as a "drinker." [17]

Height was obtained using a calibrated rod in millimeters and centimeters, with the patient barefooted and his/her back facing the wall. Weight was recorded using a digital scale (Tanita, TBF-310 GS Body Composition Analyzer, Tokyo, Japan) with the patient wearing light clothing and no shoes. Body mass index (BMI) was calculated by applying Quetelet's equation (weight/height ${ }^{2}$ ). The subjects were classified according to the following: underweight below $18.50 \mathrm{~kg} / \mathrm{m}^{2}$, normal weight between 18.50 and $24.99 \mathrm{~kg} / \mathrm{m}^{2}$, overweight (preobese) between 25.00 and $29.99 \mathrm{~kg} / \mathrm{m}^{2}$, obese class I between 30.00 and $34.99 \mathrm{~kg} / \mathrm{m}^{2}$, obese class II between 35.00 and $39.99 \mathrm{~kg} / \mathrm{m}^{2}$, and obese class III beyond $40.00 \mathrm{~kg} / \mathrm{m}^{2}$. WC was measured using calibrated measuring tapes in millimeters and centimeters, using anatomical landmarks according to the National Institutes of Health protocol: the midpoint between the lower border of the rib cage and the iliac crest, measuring at the end of expiration with participants standing and wearing only their undergarments [18]. Finally, blood pressure was determined by the auscultatory method using an adequately calibrated and validated sphygmomanometer (Tycos DS48, Welch Allyn). High blood pressure was defined according to the cutoff points proposed for metabolic syndrome in the 2009 IDF/AHA/ NHLBI/WHF/IAS/IASO consensus ( $\geq 130 / 85 \mathrm{mmHg}$ ) [19].

2.3. Laboratory Test Panel. After 8 hours of fasting, a blood sample was taken from the cubital vein and then centrifuged to obtain serum. Serum levels of glucose, total cholesterol (TC), and triacylglycerides (TAG) were determined using enzymatic colorimetric kits (HUMAN Gesellshaft Biochemica and Diagnostica $\mathrm{mbH}$ ) and a specialized computer system. The intra-assay variation coefficients for TC, TAG, and high-density lipoprotein cholesterol (HDL-C) were 3\%, 5\%, and 5\%, respectively. Insulin was determined by a double-sandwich ultrasensitive ELISA commercial kit (DRG Instruments $\mathrm{GmbH}$, Germany, Inc.). HOMA2-IR was used for IR evaluation as proposed by Levy et al. [20], and its calculation was done with the HOMA Calculator, version 2.2.2. IR was defined by a HOMA2-IR cutoff point of $\geq 2$ [21]. Serum highsensitivity C-reactive protein (hs-CRP) levels were quantified using a commercial immunoturbidimetric assay (Human Gesellshaft Biochemica and Diagnostica $\mathrm{mbH}$ ), setting the cutoff point at $\geq 0.765 \mathrm{mg} / \mathrm{L}$ [22].

2.4. Adiposity Indexes. VAI calculation was performed with the gender-specific equations proposed by Amato et al. [23] (Supplementary formula 1). LAP calculation was made applying the equation previously published by Kahn [24] (Supplementary formula 2). 
2.5. Statistical Analysis. Qualitative variables were expressed in absolute and relative frequencies, and the possible relationship between these variables was assessed with the $\chi^{2}$ test (chi-square). The difference in proportions was determined through a $Z$-test. Quantitative variables were expressed in means \pm standard deviation when there was a normal distribution and medians (interquartile range) when they had a nonnormal distribution. Student's $t$-test and Man$\mathrm{n}$-Whitney $\mathrm{U}$ test were used for normal and nonnormal distributed variables to assess arithmetic mean differences between two groups, respectively.

Receiver operating characteristic (ROC) curves were constructed to set the appropriate IR cutoff point in both the general population and according to gender for VAI and LAP. The curves were plotted using the package pROC running in $\mathrm{R}$ Project software for statistical computing, optimal cutoff points were selected using the Youden index, and the distance closest to ROC and differences between two or more AUCs were assessed through DeLong's test [25].

Multiple logistic regression models were constructed ( $95 \%$ confidence intervals) for IR presence and adjusted by age, ethnic group, education level, socioeconomic status, working status, diabetes mellitus, family history, alcohol intake, smoking habits, leisure-domain physical activity, and high blood pressure according to the IDF/AHA/NHLBI/ WHF/IAS/IASO 2009 consensus criteria. The second model adds hs-CRP. Data were analyzed using Statistical Package for the Social Sciences (SPSS) v.23 for Windows (IBM, Chicago, IL), and statistically significant results were determined when $p<0.05$.

\section{Results}

3.1. General Characteristics of the Sample. Out of the 1818 individuals studied, $51.4 \%(n=934)$ were women. The overall arithmetic mean for age was $37.7 \pm 13.9$ years, and the largest age group was the 30-59-year-old group (55.1\%; $n=1001)$. It was observed that $43.3 \%$ of the participants had IR. Table 1 depicts the general features of the studied sample.

\subsection{Adiposity Indexes and Insulin Resistance Prediction.} The LAP and VAI ROC for IR prediction in both the general population and the population distributed according to sex are shown in Figure 1. Overall, the AUC values for LAP and VAI were 0.689 (0.665-0.714) and 0.645 (0.619-0.670), respectively. On the contrary, the AUC values for LAP and VAI for women were $0.621(0.584-0.657)$ and 0.587 (0.550-0.624), respectively, whereas in men, the values were $0.759(0.728-0.791)$ for LAP and $0.704(0.670-0.738)$ for VAI. The sensitivity, specificity, Youden index, and distance to ROC are depicted in Table 2.

\subsection{Adiposity Indexes and Insulin Resistance Association.} Table 3 shows the degree of association between both adiposity indexes and IR. In the univariate analysis, the subjects located on the upper LAP tertile ( $>51.2)$ showed a higher IR frequency $(50.2 \% ; n=394)$. Likewise, the subjects in the upper VAI tertile (>2.2) have higher IR frequency (45.3\%; $n=357)$. However, in the logistic regression models, only the LAP tertiles showed a significant increase of IR risk (tertile 2: OR: 1.91; 95\% CI: $1.37-2.65 ; p<0.010$; tertile 3: OR: 5.40 ; 95\% CI: $3.48-8.39 ; p<0.01)$. This relationship remained without change after hs-CRP adjustment.

\section{Discussion}

Numerous investigations have determined that mathematical indexes such as VAI and LAP allow for the indirect evaluation of IR from visceral adiposity $[6,26,27]$. In the present study, the association and diagnostic precision of VAI and LAP in identifying IR in adults from Maracaibo city were evaluated. AUC values were more significant for LAP than for VAI. Regarding multivariate analysis, LAP proved to be a better predictor for IR presence than VAI, and therefore an index with a better discriminative power to assess IR in this population.

Since visceral adiposity is related to the development of metabolic pathologies, VAI and LAP indexes have been used as indirect and more economical predictors of T2DM, prediabetes [28], IR [29], or MS [30]. However, among the variables used to determine the visceral adiposity index, there are MS diagnosis criteria. Therefore, a correlation between them could exist [27,31], and they should not be used to diagnose SM. Likewise, other authors have used VAI and LAP to establish the presence of T2DM and prediabetes [32]. In this study, we decided to evaluate a premorbid condition such as IR, which tends to be associated with visceral adiposity and its dysfunction due to the convergence of molecular mechanisms in both metabolic disorders.

According to the ROC analysis, it was shown that LAP is the index with the greater IR predictive capacity in the general population and sex. Similar to these findings, other studies have also compared different IR surrogate indexes, reporting that the LAP index has better discrimination for the identification of IR. Fiorentino et al. performed a study of 631 individuals with different degrees of glucose intolerance who were part of the European Network on Functional Genomics of Type 2 Diabetes (EUGENE2) Project from Italy. After the ROC analysis, these researchers found that the capacity of the LAP index to identify individuals with IR defined by the AUC value $(0.728 ; 95 \%$ CI: $0.692-0.762)$ was higher than VAI (0.688; 95\% CI: 0.650-0.724), TAG/HDL-C ratio (0.693; 95\% CI: $0.646-0.729)$, and TyG index (0.688; 95\% CI: 0.650-0.723) $(p<0.05)$ [33]. Likewise, in another study performed by Mazidi et al. in 18,318 adult individuals from the NHANES data, it was found that the LAP index had a higher IR prediction capacity $(0.810$; $95 \%$ CI: $0.788-0.831)$ when compared to other traditional tools such as VAI (0.750; 95\% CI: 0.727-0.775) [34]. However, differing from the findings in our study, other researchers have reported that AUC levels for LAP and VAI were similar [11, 35-37], suggesting that both indexes have the same clinical usefulness in identifying insulin-resistant individuals. These differences in the predictive role of the visceral adiposity indexes among population groups could be due to the evolution and natural history of obesity in the subjects. 
TABLE 1: General characteristics of the sample (Maracaibo, Venezuela).

\begin{tabular}{|c|c|c|c|c|c|c|}
\hline & \multicolumn{2}{|c|}{$\begin{array}{c}\text { Without IR } \\
(n=1031)\end{array}$} & \multicolumn{2}{|c|}{ With IR $(n=787)$} & \multicolumn{2}{|c|}{ Total $(n=1818)$} \\
\hline & $n$ & $(\%)$ & $n$ & $(\%)$ & $n$ & $(\%)$ \\
\hline \multicolumn{7}{|l|}{ Gender } \\
\hline Female & 531 & 51.5 & 403 & 51.2 & 934 & 51.4 \\
\hline Male & 500 & 48.5 & 384 & 48.8 & 884 & 48.6 \\
\hline \multicolumn{7}{|l|}{ Age (years) } \\
\hline$<30$ & 394 & 38.2 & 259 & 32.9 & 653 & 35.9 \\
\hline $30-59$ & 542 & 52.6 & 459 & 58.3 & 1001 & 55.1 \\
\hline$\geq 60$ & 95 & 9.2 & 69 & 8.8 & 164 & 9.0 \\
\hline $\operatorname{BMI}\left(\mathrm{kg} / \mathrm{m}^{2}\right)(\text { mean } \pm \mathrm{SD})^{*}$ & \multicolumn{2}{|c|}{$26.3 \pm 5.2$} & \multicolumn{2}{|c|}{$30.3 \pm 6.4$} & \multicolumn{2}{|c|}{$28.0 \pm 6.1$} \\
\hline $\mathrm{WC}(\mathrm{cm})(\text { mean } \pm \mathrm{SD})^{*}$ & \multicolumn{2}{|c|}{$89.7 \pm 12.6$} & \multicolumn{2}{|c|}{$99.4 \pm 15.9$} & \multicolumn{2}{|c|}{$93.9 \pm 14.9$} \\
\hline Triglycerides $(\mathrm{mmol} / \mathrm{L})(\text { median } p 25-p 75 \text { th })^{* *}$ & \multicolumn{2}{|c|}{$1(0.7-1.5)$} & \multicolumn{2}{|c|}{$1.3(0.9-1.9)$} & \multicolumn{2}{|c|}{$1.2(0.8-1.7)$} \\
\hline HDL-C (mmol/L) (median $p 25-p 75$ th $)^{* *}$ & \multicolumn{2}{|c|}{$1.1(0.9-1.4)$} & \multicolumn{2}{|c|}{$1.1(0.9-1.2)$} & \multicolumn{2}{|c|}{$1.1(0.9-1.3)$} \\
\hline LAP (median $p 25-p 75$ th) $)^{* *}$ & \multicolumn{2}{|c|}{$27.8(14.7-49.1)$} & \multicolumn{2}{|c|}{$51.32(30.1-84.5)$} & \multicolumn{2}{|c|}{$36.1(18.7-63.5)$} \\
\hline VAI (median $p 25-p 75$ th) ${ }^{* *}$ & \multicolumn{2}{|c|}{$1.4(0.9-2.3)$} & \multicolumn{2}{|c|}{$2.1(1.3-3.2)$} & \multicolumn{2}{|c|}{$1.7(1-2.7)$} \\
\hline
\end{tabular}

IR: insulin resistance; BMI: body mass index; SD: standard deviation; WC: waist circumference; LAP: lipid accumulation product; VAI: visceral adiposity index. ${ }^{*}$ Student's $t$-test, $p<0.01 .{ }^{* *}$ Mann-Whitney $\mathrm{U}$ test, $p<0.01$.

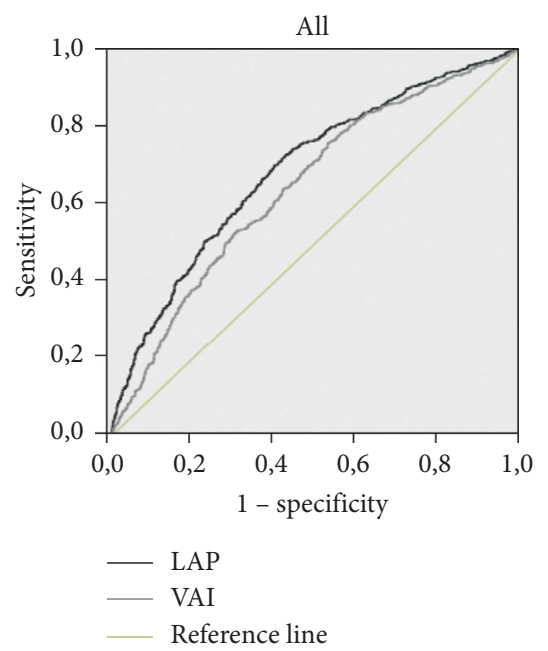

(a)

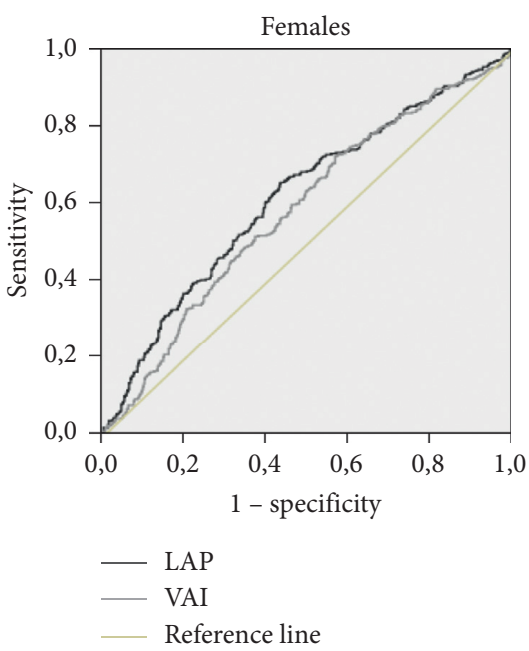

(b)

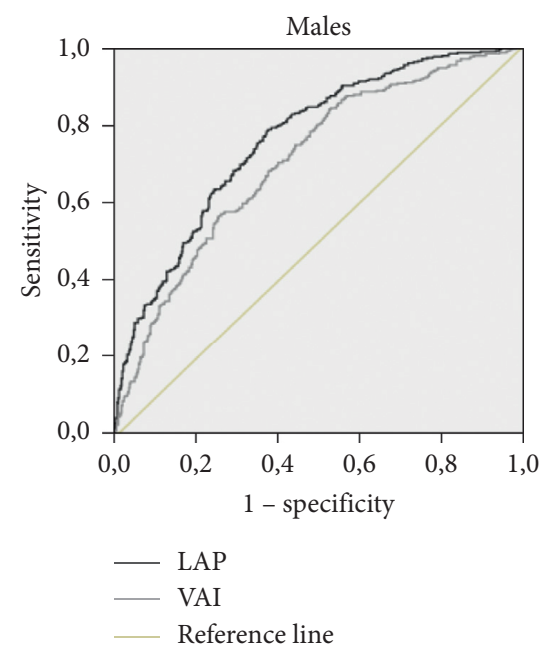

(c)

FIgURE 1: ROC curves of LAP and VAI for insulin resistance (Maracaibo, Venezuela). LAP: lipid accumulation product; VAI: visceral adiposity index.

TABLe 2: Diagnostic parameters of LAP and VAI for insulin resistance prediction (Maracaibo, Venezuela).

\begin{tabular}{|c|c|c|c|c|c|c|}
\hline & \multicolumn{2}{|c|}{ All } & \multicolumn{2}{|c|}{ Females } & \multicolumn{2}{|c|}{ Males } \\
\hline & LAP & VAI & LAP & VAI & LAP & VAI \\
\hline Cutoff points & 37.7 & 1.7 & 33.4 & 1.6 & 41.7 & 1.7 \\
\hline AUC $(95 \% \mathrm{CI})$ & $0.689(0.665-0.714)$ & $0.645(0.619-0.670)$ & $0.621(0.584-0.657)$ & $0.587(0.550-0.624)$ & $\begin{array}{c}0.759 \\
(0.728-0.791)\end{array}$ & $\begin{array}{c}0.704 \\
(0.670-0.738)\end{array}$ \\
\hline Sensitivity (\%) & 64.7 & 60.5 & 60.3 & 60.8 & 70.1 & 66.4 \\
\hline Specificity (\%) & 64.3 & 60.1 & 60.3 & 52.5 & 68.0 & 63.0 \\
\hline Youden index & 0.29 & 0.39 & 0.21 & 0.13 & 0.38 & 0.29 \\
\hline $\begin{array}{l}\text { Distance to } \\
\text { ROC }\end{array}$ & 0.50 & 0.56 & 0.56 & 0.62 & 0.44 & 0.49 \\
\hline
\end{tabular}

LAP: lipid accumulation product; VAI: visceral adiposity index. Delong's test between gender: $p<0.05$. 
TABle 3: Association between adiposity indexes and insulin resistance (Maracaibo, Venezuela).

\begin{tabular}{|c|c|c|c|c|c|c|}
\hline & \multicolumn{2}{|c|}{ Without IR } & \multicolumn{2}{|c|}{ With IR } & \multirow{2}{*}{ OR $(95 \% \mathrm{CI}) ; p^{*}$} & \multirow{2}{*}{ OR $(95 \% \mathrm{CI}) ; p^{* *}$} \\
\hline & $n$ & $(\%)$ & $n$ & $(\%)$ & & \\
\hline \multicolumn{7}{|c|}{ Lipid accumulation product } \\
\hline$<23,6$ & 444 & 43,1 & 152 & 19,3 & 1.00 & 1.00 \\
\hline $23,6-51,2$ & 354 & 34,3 & 241 & 30,6 & $1.91(1.37-2.65) ;<0.001$ & 1.91 (1.27-2.88); 0.002 \\
\hline$>51,2$ & 233 & 22,6 & 39 & 50,1 & $5.40(3.48-8.39) ;<0.001$ & $6.03(3.45-1.52) ;<0.001$ \\
\hline \multicolumn{7}{|c|}{ Visceral adiposity index } \\
\hline$<1,2$ & 430 & 41,7 & 158 & 20,1 & 1.00 & 1.00 \\
\hline $1,2-2,2$ & 337 & 32,7 & 272 & 34,6 & $1.34(0.98-1.84) ; 0.07$ & 1.35 (0.91-1.99); 0.14 \\
\hline$>2,2$ & 264 & 25,6 & 357 & 45,3 & $1.14(0.76-1.72) ; 0.53$ & $0.98(0.58-1.65) ; 0.94$ \\
\hline
\end{tabular}

Chi-square test: LAP $(176.3 ; p<0.01)$; VAI $(116.0 ; p<0.01) .{ }^{*}$ Adjusted models for gender, age, ethnic group, education level, socioeconomic status, working status, diabetes mellitus family history, alcohol intake, smoking habits, leisure-domain physical activity, and high blood pressure according to the IDF/AHA/ NHLBI/WHF/IAS/IASO 2009 consensus criteria. ${ }^{* *}$ Adjusted similar to model 1 plus hs-C-reactive protein.

Other factors involved would be the time of interaction with other cardiometabolic risk factors, the differences in the frequency of these risk factors in different populations, and the influence of ethnicity and genetic considerations.

Alternatively, although higher LAP and VAI levels were associated with a higher IR frequency, only high LAP levels showed a significantly higher risk of presenting IR. These findings were similar to those reported by $\mathrm{Oh}$ et al., who demonstrated that high LAP levels were associated with higher HOMA-IR levels in healthy Korean women [38]. Likewise, a cross-sectional study, including 2,524 nondiabetic Chinese subjects, found that HOMA-IR scores significantly rose as LAP increased [39]. Different authors have also shown a higher positive correlation between LAP and HOMA-IR compared to VAI [33, 37, 39]. In this sense, Mazidi et al. reported a significantly higher positive correlation between HOMA-IR and LAP $(r=0.551)$ compared to other indexes such as the TyG index $(r=0.502)$, apVAT $(r=0.454)$, TAG:HDL-C ratio $(r=0.441)$, and VAI $(r=0.451)$ [34]. These results were similar to those reported by Xia et al. in which HOMA-IR was positively correlated to LAP $(r=0.361)$, WC $(r=0.347)$, and IMC $(r=0.346)$. In the same study, through a multivariant logistic regression, it was shown that LAP has a higher impact on HOMA-IR scores compared to BMI in men (standardized coefficient $\beta=0.22, p<0.001$ vs. standardized coefficient $\beta=0.20$, $p<0.001$ ) and women (standardized coefficient $\beta=0.24$, $p<0.001$ vs. standardized coefficient $\beta=0.15, p<0.001$ ) [37]. However, VAI was not included in this analysis. Similarly, other studies have highlighted the superiority of LAP in contrast to other indexes used in individuals with T2DM [26], MS, and cardiovascular disease [40] and women with PCOS [29].

These findings could be explained since both consider $\mathrm{WC}$ as an anthropometric parameter of central obesity. They also include concentrations of proatherogenic lipids (TAG in the case of LAP) and antiatherogenic lipids (HDL-C in the case of VAI), which together would allow for the evaluation of adiposity status and to indirectly estimate the metabolic profile of the individuals [41]. IR leads to hypertriglyceridemia due to the inability of insulin to inhibit lipid production at the hepatic level [42, 43]. Furthermore, hypertriglyceridemia has been associated with the ectopic deposit and accumulation of lipids in visceral tissue [44, 45]. Meanwhile, although WC does not distinguish between subcutaneous and visceral tissue, it has been strongly associated with higher cardiometabolic risk [46]. In this sense, visceral adipose tissue has a higher rate of lipolysis and proinflammatory adipokines' secretion, promoting a lowgrade inflammation state, which alters insulin signalling [47-49]. By including parameters associated with metabolic disturbances associated with deflective insulin action, LAP positions itself as a better IR predictor [50].

Our research has some limitations, among which it is essential to highlight the cross-sectional nature of the study, which avoids the establishment of relationships of causality among the indexes. Additionally, instead of using the gold standard in evaluating IR (hyperinsulinemic-euglycemic clamp technique), HOMA2-IR was used, which is a tool validated in different populations, especially in epidemiologic analyses in low-resource contexts [50].

In conclusion, LAP shows a higher predictive capacity and association with IR in the Maracaibo city population than VAI and could be an easy and nonexpensive tool that can be used in low-income settings.

\section{Data Availability}

The excel template data used to support the findings of this study have been deposited in the F1000 research repository (10.5256/f1000research.14571.d201851).

\section{Conflicts of Interest}

The authors declare no conflicts of interest.

\section{Supplementary Materials}

Supplementary formula 1: visceral adiposity index formula. TAG and HDL-C are expressed in mmol/L. VAI: visceral adiposity index; WC: waist circumference; BMI: body mass index; TAG: triacylglycerides; HDL-C: high-density lipoprotein cholesterol. Supplementary formula 2: lipid accumulation product formula. TAG are expressed in $\mathrm{mmol} / \mathrm{L}$. LAP: lipid accumulation product; WC: waist circumference; TAG: triacylglycerides. (Supplementary Materials) 


\section{References}

[1] J. Rojas, V. Bermúdez, E. Leal et al., "Insulinorresistencia e hiperinsulinemia como factores de riesgo para enfermedad cardiovascular," International Journal of Diabetes in Developing Countries, vol. 2, no. 3, pp. 53-64, 2012.

[2] R. Liu, K. K. Christoffel, W. J. Brickman et al., "Do static and dynamic insulin resistance indices perform similarly in predicting pre-diabetes and type 2 diabetes?" Diabetes Research and Clinical Practice, vol. 105, no. 2, pp. 245-250, 2014.

[3] R. A. DeFronzo, J. D. Tobin, and R. Andres, "Glucose clamp technique: a method for quantifying insulin secretion and resistance," American Journal of Physiology-Endocrinology and Metabolism, vol. 237, no. 3, pp. E214-E223, 1979.

[4] J. Otten, B. Ahrén, and T. Olsson, "Surrogate measures of insulin sensitivity vs the hyperinsulinaemic-euglycaemic clamp: a meta-analysis," Diabetologia, vol. 57, no. 9, pp. 1781-1788, 2014.

[5] B. Ji, H. Qu, H. Wang, H. Wei, and H. Deng, "Association between the visceral adiposity index and homeostatic model assessment of insulin resistance in participants with normal waist circumference," Angiology, vol. 68, no. 8, pp. 716-721, 2017.

[6] V. J. Bermúdez, J. Salazar, R. Añez et al., "Optimal cutoff for visceral adiposity index in a Venezuelan population: results from the Maracaibo city metabolic syndrome prevalence study," Revista Argentina de Endocrinología y Metabolismo, vol. 54, no. 4, pp. 176-183, 2017.

[7] P. Mathieu, P. Poirier, P. Pibarot, I. Lemieux, and J.-P. Després, "Visceral obesity: the link among inflammation, hypertension, and cardiovascular disease," Hypertension (Dallas, Tex: 1979), vol. 53, no. 4, pp. 577-584, 2009.

[8] D. Dai, Y. Chang, Y. Chen et al., "Visceral adiposity index and lipid accumulation product index: two alternate body indices to identify chronic kidney disease among the rural population in northeast China," International Journal of Environmental Research and Public Health, vol. 13, no. 12, p. 1231, 2016.

[9] J. Wu, L. Gong, Q. Li et al., "A novel visceral adiposity index for prediction of type 2 diabetes and pre-diabetes in Chinese adults: a 5 year prospective study," Scientific Reports, vol. 7, no. 1, p. 13784, 2017.

[10] Z. Biyik and I. Guney, "Lipid accumulation product and visceral adiposity index: two new indices to predict metabolic syndrome in chronic kidney disease," European Review for Medical and Pharmacological Sciences, vol. 23, no. 5, pp. 2167-2173, 2019.

[11] X. Huang, Q. Wang, T. Liu et al., "Body fat indices as effective predictors of insulin resistance in obese/non-obese polycystic ovary syndrome women in the Southwest of China," Endocrine, vol. 65, no. 1, pp. 81-85, 2019.

[12] V. Bermúdez, R. P. Marcano, C. Cano et al., "The Maracaibo city metabolic syndrome prevalence study: design and scope," American Journal of Therapeutics, vol. 17, no. 3, pp. 288-294, 2010.

[13] J. Rojas, V. Bermudez, R. Añez et al., "Comportamiento Epidemiológico del síndrome metabólico en el municipio Maracaibo-Venezuela," Sindrome Cardiometabólico., vol. 3, no. 2, pp. 31-42, 2013.

[14] H. Méndez Castellano and M. de Méndez, "Estratificación social y biología humana: método Graffar modificado," Arch Venez Pueric Pediatrics, vol. 49, no. 3, pp. 93-104, 1986.

[15] V. Bermúdez, "Smoking habit in adult population from Maracaibo city, Venezuela," International Journal of Respiratory and Pulmonary Medicine, vol. 3, no. 4, p. 61, 2016.
[16] Guidelines for Data Processing and Analysis of the International Physical Activity Questionnaire (IPAQ), 2005, http://www. IPAQ.ki.sehttps://www.researchgate.net/publication/267932370_ Guidelines_for_data_processing_and_analysis_of_the_ International_Physical_Activity_Questionnaire_IPAQ2005_ URL_httpwwwIPAQkise.

[17] V. Bermúdez, Y. Torres, V. Apruzzese et al., "Alcohol drinking patterns in the adult population from the Maracaibo municipality, Zulia-Venezuela," Revista Latinoamericana de Hipertension, vol. 9, no. 1, pp. 21-28, 2014.

[18] Health Statistics, NHANES III Reference Manuals and Reports (CDROM), Centers for Disease Control and Prevention, Atlanta, GA, USA, 1996, https://wwwn.cdc.gov/nchs/data/ nhanes $3 /$ manuals/.

[19] K. G. M. M. Alberti, R. H. Eckel, S. M. Grundy et al., "Harmonizing the metabolic syndrome: a joint interim statement of the international diabetes federation task force on epidemiology and prevention; national heart, lung, and blood institute; American heart association; world heart federation; international atherosclerosis society; and international association for the study of obesity," Circulation, vol. 120, no. 16, pp. 1640-1645, 2009.

[20] J. C. Levy, D. R. Matthews, and M. P. Hermans, "Correct homeostasis model assessment (HOMA) evaluation uses the computer program," Diabetes Care, vol. 21, no. 12, pp. 2191-2192, 1998.

[21] V. Bermúdez, J. Rojas, M. S. Martínez et al., "Epidemiologic behavior and estimation of an optimal cut-off point for homeostasis model assessment-2 insulin resistance: a report from a Venezuelan population," International Scholarly Research Notices, vol. 2014, Article ID 616271, 10 pages, 2014.

[22] V. Bermúdez, M. Cabrera, L. Mendoza et al., "High-sensitivity c-reactive protein epidemiological behavior in adult individuals from Maracaibo, Venezuela," Revista Latinoamericana de Hipertension, vol. 8, no. 1, pp. 22-29, 2015.

[23] M. C. Amato, C. Giordano, M. Galia et al., "Visceral adiposity index: a reliable indicator of visceral fat function associated with cardiometabolic risk," Diabetes Care, vol. 33, no. 4, pp. 920-922, 2010.

[24] H. S. Kahn, "The "lipid accumulation product" performs better than the body mass index for recognizing cardiovascular risk: a population-based comparison," BMC Cardiovascular Disorders, vol. 5, no. 1, p. 26, 2005.

[25] X. Robin, N. Turck, A. Hainard et al., "pROC: an open-source package for R and S+ to analyze and compare ROC curves," BMC Bioinformatics, vol. 12, no. 1, p. 77, 2011.

[26] H. S. Kahn, "The lipid accumulation product is better than BMI for identifying diabetes: a population-based comparison,” Diabetes Care, vol. 29, no. 1, pp. 151-153, 2006.

[27] S. Pekgor, C. Duran, U. Berberoglu, and M. A. Eryilmaz, "The role of visceral adiposity index levels in predicting the presence of metabolic syndrome and insulin resistance in overweight and obese patients," Metabolic Syndrome and Related Disorders, vol. 17, no. 5, pp. 296-302, 2019.

[28] C. M. de Oliveira, J. Pavani, J. E. Krieger, R. de Oliveira Alvim, C. A. Mourão-Junior, and A. da Costa Pereira, "Body adiposity index in assessing the risk of type 2 diabetes mellitus development: the baependi heart study," Diabetology \& Metabolic Syndrome, vol. 11, no. 1, p. 76, 2019.

[29] G. A. Abruzzese, G. E. Cerrrone, J. M. Gamez et al., "Lipid accumulation product (LAP) and visceral adiposity index (VAI) as markers of insulin resistance and metabolic associated disturbances in young argentine women with polycystic ovary syndrome," Hormone and Metabolic 
Research = Hormon - and Stoffwechselforschung = Hormones et Metabolisme, vol. 49, no. 1, pp. 23-29, 2017.

[30] K. Baveicy, S. Mostafaei, M. Darbandi, B. Hamzeh, F. Najafi, and Y. Pasdar, "Predicting metabolic syndrome by visceral adiposity index, body roundness index and a body shape index in adults: a cross-sectional study from the Iranian RaNCD cohort data," Diabetes, Metabolic Syndrome and Obesity: Targets and Therapy, vol. 13, pp. 879-887, 2020.

[31] M. E. Zaki, S. Kamal, H. Reyad et al., "The validity of body adiposity indices in predicting metabolic syndrome and its components among Egyptian women," Open Access Macedonian Journal of Medical Sciences, vol. 4, no. 1, pp. 25-30, 2016.

[32] N. Ahn, S. E. Baumeister, U. Amann et al., "Visceral adiposity index (VAI), lipid accumulation product (LAP), and product of triglycerides and glucose (TyG) to discriminate prediabetes and diabetes," Scientific Reports, vol. 9, no. 1, p. 9693, 2019.

[33] T. V. Fiorentino, M. A. Marini, E. Succurro, F. Andreozzi, and G. Sesti, "Relationships of surrogate indexes of insulin resistance with insulin sensitivity assessed by euglycemic hyperinsulinemic clamp and subclinical vascular damage," BMJ Open Diabetes Research \& Care, vol. 7, no. 1, Article ID e000911, 2019.

[34] M. Mazidi, A.-P. Kengne, N. Katsiki, D. P. Mikhailidis, and M. Banach, "Lipid accumulation product and triglycerides/ glucose index are useful predictors of insulin resistance," Journal of Diabetes and its Complications, vol. 32, no. 3, pp. 266-270, 2018.

[35] B. Kim, H. Y. Choi, W. Kim et al., "The cut-off values of surrogate measures for insulin resistance in the Korean population according to the Korean genome and epidemiology study (KOGES)," PLoS One, T. Muka, Ed., vol. 13, no. 11, Article ID e0206994, 2018.

[36] L.-K. Er, S. Wu, H.-H. Chou et al., "Triglyceride glucose-body mass index is a simple and clinically useful surrogate marker for insulin resistance in nondiabetic individuals," PLoS One, vol. 11, no. 3, Article ID e0149731, 2016.

[37] G. Anik Ilhan, B. Yildizhan, and T. Pekin, "The impact of lipid accumulation product (LAP) and visceral adiposity index (VAI) on clinical, hormonal and metabolic parameters in lean women with polycystic ovary syndrome," Gynecological Endocrinology, vol. 35, no. 3, pp. 233-236, 2019.

[38] J.-Y. Oh, Y.-A. Sung, and H. J. Lee, “The lipid accumulation product as a useful index for identifying abnormal glucose regulation in young Korean women," Diabetic Medicine, vol. 30, no. 4, pp. 436-442, 2013.

[39] C. Xia, R. Li, S. Zhang et al., "Lipid accumulation product is a powerful index for recognizing insulin resistance in nondiabetic individuals," European Journal of Clinical Nutrition, vol. 66, no. 9, pp. 1035-1038, 2012.

[40] M. Velasco, "Lipid accumulation product (LAP) and visceral adiposity index (VAI) a simple and clinically useful surrogate marker for metabolic syndrome and cardiovascular disease predictor in non-diabetic Venezuelan adults," Journal of Pharma \& Pharmaceutical Sciences, vol. 2, no. 1, pp. 1-8, 2017.

[41] T. Du, G. Yuan, M. Zhang, X. Zhou, X. Sun, and X. Yu, "Clinical usefulness of lipid ratios, visceral adiposity indicators, and the triglycerides and glucose index as risk markers of insulin resistance," Cardiovascular Diabetology, vol. 13, no. 1, p. 146, 2014.

[42] J. D. Sparks, C. E. Sparks, and K. Adeli, "Selective hepatic insulin resistance, VLDL overproduction, and hypertriglyceridemia," Arteriosclerosis, Thrombosis, and Vascular Biology, vol. 32, no. 9, pp. 2104-2112, 2012.
[43] D. Santoleri and P. M. Titchenell, "Resolving the paradox of hepatic insulin resistance," Cellular and Molecular Gastroenterology and Hepatology, vol. 7, no. 2, pp. 447-456, 2019.

[44] M. Aslam, S. Aggarwal, K. K. Sharma, V. Galav, and S. V. Madhu, "Postprandial hypertriglyceridemia predicts development of insulin resistance glucose intolerance and type 2 diabetes," PLoS One, vol. 11, no. 1, Article ID e0145730, 2016.

[45] X. Bi, Y. T. Loo, and C. J. Henry, "Ultrasound measurement of intraabdominal fat thickness as a predictor of insulin resistance and low HDL cholesterol in Asians," Nutrition, vol. 5556, pp. 99-103, 2018.

[46] S. Klein, D. B. Allison, S. B. Heymsfield et al., "Waist circumference and cardiometabolic risk: a consensus statement from shaping America's health: association for weight management and obesity prevention; NAASO, the obesity society; the American society for nutrition; and the American diabetes association," Diabetes Care, vol. 30, no. 6, pp. 1647-1652, 2007.

[47] C. Zou and J. Shao, "Role of adipocytokines in obesity-associated insulin resistance," The Journal of Nutritional Biochemistry, vol. 19, no. 5, pp. 277-286, 2008.

[48] X.-F. Zhuang, M.-M. Zhao, C.-L. Weng, and N.-L. Sun, "Adipocytokines: a bridge connecting obesity and insulin resistance," Medical Hypotheses, vol. 73, no. 6, pp. 981-985, 2009.

[49] K. Verboven, K. Wouters, K. Gaens et al., "Abdominal subcutaneous and visceral adipocyte size, lipolysis and inflammation relate to insulin resistance in male obese humans," Scientific Reports, vol. 8, no. 1, p. 4677, 2018.

[50] M. D. Jensen, "Adipose tissue as an endocrine organ: implications of its distribution on free fatty acid metabolism," European Heart Journal Supplements, vol. 8, no. suppl_B, 2006. 